\title{
A Radiometeorological Study, Part III. A New Turbulence Parameter
}

\author{
B. R. Bean, E. J. Dutton, \\ J. A. Lane, and W. B. Sweezy \\ Contribution from Central Radio Propagation Laboratory, National Bureau of Standards, Boulder, Colo.
}

(Received June 7, 1963)

\begin{abstract}
The concept of thermal stability is utilized to derive expressions for the radio refractivity of an air parcel undergoing adiabatic compression or expansion. These expressions are of exponential form with scale heights of $12.5 \mathrm{~km}$ for the dry adiabatic process and $7.0 \mathrm{~km}$ for the wet adiabatic process. The adiabatic curves for $N$ are determined solely from conditions at the earth's surface. A new turbulence parameter, $\Pi$, is derived as the difference of the environmental refractive index structure and the adiabatic curves for an air parcel lifted from the surface to $3 \mathrm{~km}$. This parameter yields correlations with three hourly median field strength data that are not statistically different from those obtained with the much simpler parameter, $\Delta N$. The correlations obtained with the equivalent gradient are not statistically different from zero.
\end{abstract}

\section{Introduction}

We shall here attempt to unify past work utilizing the surface value of the refractive index and the concept of atmospheric stability. To do so we shall first review the concepts of atmospheric stability from the viewpoint of temperature structure. In the process we shall show that past efforts have neglected the important role of the conditions at the earth's surface, and then we shall extend this work to derive an analogous expression for the radio refractive index.

\section{Concept of Thermal Stability}

The stratification of the earth's atmosphere by and large reflects the control of the earth's gravitational field. This average structure is, however, constantly disturbed by the intrusion of thermal plumes of heated air rising from the differentially heated surface of the earth as well as the mechanical mixing produced by the passage of air currents. It is customary to assume that parcels of air are forced upwards through the normal stratified atmosphere without mixing with the environmental air mass. Since the parcel is forced to rise, be it by convection or mechanical mixing, it is also assumed that it expands and cools without exchange of heat; i.e., adiabatically. In such a process it can be shown that the pressure, $P$, is given by

$$
P=P_{0}\left(\frac{T}{T_{0}}\right)^{g / R \alpha}
$$

where $T$ is the temperature $\left({ }^{\circ} \mathrm{K}\right), g$, the acceleration of gravity; $R$, the gas constant for air; and $\alpha$, the constant lapse of temperature with height is

$$
-\alpha=+9.8^{\circ} \mathrm{C} / \mathrm{km} \text {. }
$$

The zero subscripts in (1) refer to conditions at the earth's surface. Equations (1) and (2) are taken to hold as long as saturation does not occur. It is further assumed that the mixing ratio

$$
e \cong e_{0} \frac{P}{P_{0}}
$$

is constant throughout the process. In (3) $e$ is the partial pressure of water vapor.

A parcel of air forced to rise will cool to the dewpoint if forced aloft far enough. Further rising will lead to condensation. Conversely a parcel of air which sinks down will have work done upon it adiabatically and will become warmer than it was in its original elevated position. Air following a condensation curve has a value of $\alpha, \alpha^{*}$, of $6^{\circ} \mathrm{C} / \mathrm{km}$. In addition, as water vapor condenses out of the parcel, the vapor pressure will decrease. We here assume that the vapor pressure will follow that of the saturation vapor pressure curve, $e_{s}$, as given by

$$
e_{s z}=e_{s l} \exp \left[-\beta \alpha^{*}(z-l)\right], \quad z \geq l,
$$

where $l$ is the height of the lift condensation level and $\beta=0.064\left({ }^{\circ} \mathrm{C}\right)^{-1}$. The value of $l$ is given [Hewson and Longley, 1944] with sufficient accuracy for practical applications by 


$$
l \cong 0.125\left(T-T_{d}\right)_{0}(\mathrm{~km})
$$

where the zero subscript indicates that the difference between the temperature and the dewpoint, $T_{d}$, need be evaluated only at the earth's surface.

Consider now a parcel rising through some environmental distribution of temperature. The dry adiabatic lapse of temperature with height is greater than that observed on the average in the atmosphere $\left(6{ }^{\circ} \mathrm{C} / \mathrm{km}\right)$ and thus the parcel becomes cooler, and more dense, than the environmental air and will sink to its initial conditions with the removal of the lifting force. If, on the contrary, anywhere in its trajectory it becomes warmer, and thus less dense, it will become unstable and rise of its own buoyancy through the environmental air. Past radiometeorological studies have taken the area between the adiabatic curves and the environmental temperature distribution on a pressure ( $\sim$ height) versus temperature chart as a measure of atmospheric stability. On the general argument that the effects of atmospheric stability, or turbulence, influence radio waves only through the radio refractive index, we shall extend the above concepts to the radio refractive index.

\section{Adiabatic Lapses of $N$}

The radio refractivity, with the usual units, is given by

$$
N=\underset{T}{77.6}\left(P+\frac{4810 e}{T}\right)
$$

Introducing the dry adiabatic variations of $P, T$, and $e$ as given above one obtains the dry adiabatic decrease, $N_{d}$, of $N$ :

$$
N_{d}=N_{0}\left(1-\frac{\alpha z}{T_{0}}\right)^{\frac{\mathrm{g}}{R \alpha}-2}\left\{1-\frac{77.6 P_{0} \alpha_{z}}{T_{0}^{2} N_{0}}\right\}
$$

which is valid for $z \leq l$. The distribution of $N_{d}$ with $z$ may be evaluated by taking the logarithms of both sides of (7),

$\ln N_{d}=\ln N_{0}+\left(\frac{g}{R \alpha}-2\right) \ln \left(1-\frac{\alpha z}{T_{0}}\right)+\ln \left(1-\frac{77.6 P_{0} \alpha_{z}}{T_{0}^{2} N_{0}}\right)$

and expanding the logarithms on the right-hand side (r.h.s.) of (8) with the neglection of second order terms where appropriate for our applications of $z \leq 3 \mathrm{~km}$ :

or

$$
\ln N_{d} \simeq \ln N_{0}-\Gamma_{d} z
$$

where

$$
N_{d} \simeq N_{0} \exp \left(-\Gamma_{d} z\right)
$$

$$
\Gamma_{d}=\frac{g}{R T_{0}}-\frac{2 \alpha}{T_{0}}+\frac{77.6 P_{0} \alpha}{N_{0} T_{0}^{2}} .
$$

When evaluated for the standard conditions of $T_{0}=288^{\circ} \mathrm{K}\left(15^{\circ} \mathrm{C}\right)$ and $P_{0}=1013.2 \mathrm{mb}, \Gamma_{d}$ becomes

$$
\Gamma_{d}=0.08(\mathrm{~km})^{-1} \text {. }
$$

It is sometimes more convenient to use the dry adiabatic scale height, $H_{d}$;

$$
H_{d}=\frac{1}{\Gamma_{d}}=12.5 \mathrm{~km} .
$$

Above the local condensation level, the expression for $N$ becomes that of the wet adiabatic, $N_{w}$ :

$$
\begin{aligned}
N_{w}=\left(1+\frac{\alpha^{*} z}{T_{l}}\right)^{-2}\left\{D_{l}\left(1+\frac{\alpha z}{T_{l}}\right)^{\frac{g}{R \alpha *}+1}\right. \\
\left.+W_{l} \exp \left(\beta \alpha^{*} z\right)\right\}
\end{aligned}
$$

where, for convenience

$$
D_{l}=77.6 \frac{P_{l}}{T_{l}} \text { and } W_{l}=\frac{77.6(4810) e_{s l}}{T_{l}^{2}} .
$$

(Throughout this development the subscript $l$ refers to the local condensation level.) Applying the same approximations as in the derivation of (9) one obtains

where

$$
\ln N_{w}=\ln N_{l}-\Gamma_{w} z
$$

$$
\Gamma_{w}=\frac{2 \alpha^{*}}{T_{l}}+\frac{\left(\frac{g}{R \alpha^{*}}+1\right) \alpha^{*} D_{l}}{T_{l} N_{l}}+\frac{W_{l}}{N_{l}} \beta \alpha^{*} .
$$

For the standard conditions assumed above and $e_{0}$ corresponding to 60 percent relative humidity $H_{w}$ $\sim 7.5 \mathrm{~km}$. Again, the form of the wet adiabatic lapse of $N$ is given by

$$
N_{w}=N_{l} \exp \left(-\Gamma_{w} z\right), \quad z \geq l .
$$

We shall now apply these results to the derivation of a refractive index turbulence parameter.

\section{Turbulence Parameter, II}

Analogously to the concept of thermal stability, we define II as the area between the environmental $N(z)$ curve and the appropriate adiabatic decrease of $N$. That is

$$
\Pi=\int_{0}^{3 \mathrm{~km}}\left(N_{\text {observed }}-N_{\text {adiabatic }}\right) d z
$$

where the integration is arbitrarily taken from the surface to $3 \mathrm{~km}$. This then extends the integration well above the crossover height of the radio horizon rays from transmitter and receiver normally encountered in tropospheric propagation. The integral for $\Pi$ may be written

$$
\begin{aligned}
\Pi=\int_{0}^{3} N d z-\int_{0}^{l} N_{0} & \exp \left(-\Gamma_{d} z\right) d z \\
& -\int_{l}^{3} N_{l} \exp \left[-\Gamma_{w}(z-l)\right] d z .
\end{aligned}
$$


Noting that

$$
N_{l} \equiv N_{0} \exp \left(-\Gamma_{d} l\right),
$$

(19) then becomes, upon integration,

$$
\begin{aligned}
\Pi=\int_{0}^{3} N d z & +\frac{N_{d}}{\Gamma_{d}}\left[\exp \left(-\Gamma_{d} l\right)-1\right] \\
& +\frac{N_{0} \exp \left(-\Gamma_{d} l\right)}{\Gamma_{w}}\left[\exp \left(-\Gamma_{w}(3-l)-1\right] .\right.
\end{aligned}
$$

$\Pi$ is thus dependent upon both $N$ profile characteristics and initial conditions. In fact, all the terms on the r.h.s. of (20) save

$$
\int_{0}^{3} N d z
$$

are determined from the conditions at the earth's surface. One might expect, then, that $\Pi$ would constitute a useful radiometeorological parameter since it includes the parameter $N_{0}$, the concept of atmospheric stability, and the integrated $N$-profile characteristics. We shall now apply this new parameter to the radio data presented in detail in part II. The values of $H_{d}$ and $H_{w}$ utilized are those for standard sea-level conditions; i.e., $12.5 \mathrm{~km}$ and $7.5 \mathrm{~km}$ respectively.

\section{Comparison of Radiometeorological Parameters}

The various radiometeorological parameters discussed in part I plus $\Pi$ as derived from the Rantoul and Joliet radiosondes were tested for their relative utility by comparison with the radio data of WBKBTV and WENR-TV (see table 2, part II). Correlation coefficients between the radio data (three hourly medians) of each path and the various radiometeorological parameters were determined for each of the profile categories given in part II, table 3 , as well as for all available data. It was not possible to fit all of the data into the various categories since these were deliberately made very strict in order to explore the utility of each parameter within each of the propagation mechanisms assumed for the profile categories (pt. II, table 1). Thus it is observed that the sum of the individual categories does not equal all available data. The results of these correlations are given in table 1 . Before discussing these results it is well to note that the crossover height of the tangent rays from each end of the path were calculated by detailed ray tracing [Bean and Thayer, 1959] for each radiosonde ascent. The values as determined from the Rantoul and Joliet soundings were averaged. This average crossover height was then used to determine the values of $\Pi$ below the crossover height $\left(\Pi^{\prime \prime}\right)$, above the crossover height $\left(\Pi^{\prime}\right)$, and $g_{e}$. As listed in table $1, \Pi$ represents the value of (18) for the height increment zero to $3 \mathrm{~km}$.

The conclusions reached below are based only upon the data of category six (all data) of table 1 ; the sample size of the individual categories sometimes being so small as to prohibit the attachment of any significance to the observed results. It is thus evident from table 1 that the results obtained with $I$ are comparable with those using $\Pi^{\prime}$, due supposedly to the crossover heights being on the order of $100 \mathrm{~m}$ for these paths. $\Pi^{\prime \prime}$ appears to contribute little to the final value of $I$ and is poorly correlated with the radio data. The overall conclusion that one reaches from the data of table 1 is that the most promising of the parameters considered is $\Pi$ closely followed by $\Delta N$ and $N_{s}$. In fact, the difference between the results obtained with $\Pi$ and $\Delta N$ gives one pause to consider if the added complexity of determining $\Pi$ is worth the relatively small gain in correlation with the radio data. Further, examination of the 95 percent confidence belts of the correlations [Crow, Davis, and Maxfield, 1960] shows that the results obtained with $\Delta N$ and $\Pi$ are not statistically different within this confidence band. Similarly, it is concluded

\begin{tabular}{|c|c|c|c|c|c|c|c|c|c|}
\hline \multirow{2}{*}{ Station } & \multirow{2}{*}{ Category* } & \multirow{2}{*}{$\begin{array}{l}\text { Sample } \\
\text { size }\end{array}$} & \multicolumn{7}{|c|}{ Radio meteorological variable } \\
\hline & & & II & $\Pi^{\prime}$ & $\Pi^{\prime \prime}$ & $g_{e}$ & $g_{o}$ & $N_{s}$ & $\triangle N$ \\
\hline $\begin{array}{l}\text { WENR-TV }(179.75 \\
\mathrm{Mc} / \mathrm{s})\end{array}$ & $\begin{array}{l}1 \\
2 \\
3 \\
4 \\
5 \\
6\end{array}$ & $\begin{array}{r}68 \\
21 \\
101 \\
6 \\
196 \\
518\end{array}$ & $\begin{array}{r}-0.379 \\
-.206 \\
-.175 \\
-.363 \\
-.566 \\
-.462\end{array}$ & $\begin{array}{r}-0.380 \\
-.268 \\
-.175 \\
-.350 \\
-.573 \\
-.460\end{array}$ & $\begin{array}{r}-0.186 \\
.200 \\
.009 \\
-.432 \\
-.031 \\
-.017\end{array}$ & $\begin{array}{r}0.189 \\
.004 \\
.029 \\
.499 \\
.056 \\
.072\end{array}$ & $\begin{array}{r}-0.188 \\
-.135 \\
-.030 \\
-.415 \\
-.177 \\
-.176\end{array}$ & $\begin{array}{r}0.141 \\
.053 \\
-.094 \\
.090 \\
.387 \\
.289\end{array}$ & $\begin{array}{r}-0.346 \\
-.265 \\
-.455 \\
.087 \\
-.564 \\
-.454\end{array}$ \\
\hline $\begin{array}{l}\text { WBKB-TV }(71.75 \\
\mathrm{Mc} / \mathrm{s})\end{array}$ & $\begin{array}{l}1 \\
2 \\
3 \\
4 \\
5 \\
6\end{array}$ & $\begin{array}{r}67 \\
27 \\
113 \\
6 \\
213 \\
564\end{array}$ & $\begin{array}{r} \\
-0.295 \\
-.207 \\
-.269 \\
-.244 \\
-.552 \\
-.438\end{array}$ & $\begin{array}{r}-0.298 \\
-.184 \\
-.271 \\
-.220 \\
-.556 \\
-.440\end{array}$ & $\begin{array}{r}-0.223 \\
-.138 \\
.076 \\
-.685 \\
-.058 \\
.009\end{array}$ & $\begin{array}{r}-0.093 \\
-.104 \\
.165 \\
.308 \\
.062 \\
.043\end{array}$ & $\begin{array}{r}0.079 \\
.094 \\
-.081 \\
-.369 \\
-.115 \\
-.103\end{array}$ & $\begin{array}{l}0.011 \\
.168 \\
.070 \\
.257 \\
.417 \\
.213\end{array}$ & $\begin{array}{r}-0.169 \\
-.161 \\
-.349 \\
.348 \\
-.464 \\
-.350\end{array}$ \\
\hline
\end{tabular}
that $g_{e}$ yields correlations not statistically different from zero.

TABLE 1. Summary of correlations

*Category

$1=$ Unstratified.

$2=$ Ground based duct.

$3=$ Elevated layer.

$4=$ Subrefractive layer.

$6=$ All data for period of record. 
The above data also allows one to evaluate a parameter of the form

$$
H_{1}=a g_{e}+b \Pi^{\prime}
$$

which is analogous to Misme's recently suggested $M$ parameter [Misme, 1960]. The parameter $H_{1}$ given above is physically more realistic for radio purposes since it incorporates a measure of the refractive index turbulence rather than thermal stability. The correlations obtained between field strength and $H_{1}$ are

\section{WENR-TV: 0.46 \\ WBKB-TV: 0.44}

which represents, for the significant figures carried, no improvement over the use of $\Pi$ alone. This last analysis suggests that it might be desirable to consider other combinations such as

$$
H_{2}=a_{2} \Delta N+b_{2} \Pi \text {. }
$$

The correlations for this case are

$\begin{array}{ll}\text { WENR-TV: } & 0.49 \\ \text { WBKB-TV: } & 0.44\end{array}$

which represents a slight improvement over $\Pi$ alone.

\section{Conclusions}

In conclusion, an atmospheric stability parameter, $\Pi$, based upon the radio refractive index has been developed. The $\Pi$ parameter, like thermal stability, is an integrated effect and thus many combinations of vertical temperature and humidity distributions may yield the same value of $\Pi$ (the same is true of thermal stability). Nevertheless, the correlation obtained between $\Pi$ and radio data on a three hourly median basis is perhaps the most encouraging obtained to date but does not yield correlations significantly different from those obtained with the much simpler parameter, $\Delta N$. This conclusion could well change with broader experience on other paths and radio frequencies.

\section{References}

Bean, B. R., and G. D. Thayer (1959), On models of the atmospheric refractive index, Proc. IRE 47, No. 5, 740-755.

Crow, E. L., F. A. Davis, M. W. Maxfield (1960), Statistics Manual, pp. 159 and 279 (Dover Publications, Inc., New York).

Hewson, E. W., and R. W. Longley (1944), Meteorology, Theoretical and Applied, p. 352 (John Wiley \& Sons, Inc., New York, N.Y.).

Misme, P. (1960), L'influence du gradient equivalent et de la stabilite atmospherique dans les liaisons transhorizon au Sahara et au Congo, Ann. Telecomm. 16, 110.

(Paper 67D6-293) 